\title{
Planning stability in a product recovery system
}

\section{Planungsstabilität in einem Lagerhaltungsmodell mit Güterrückflüssen}

\author{
Gerald Heisig $^{1}$ and Moritz Fleischmann ${ }^{2}$ \\ 1 Faculty of Economics and Management, University of Magdeburg, P.O. Box 4120, \\ 39016 Magdeburg, Germany (e-mail: gerald.heisig@ww.uni-magdeburg.de) \\ 2 Faculty of Business Administration, Erasmus University Rotterdam, P.O. Box 1738, \\ 3000 DR Rotterdam, Netherlands (e-mail: MFleischmann@fac.fbk.eur.nl)
}

\begin{abstract}
Recovery of used products is an issue of growing importance due to customer expectations and environmental regulation. As a consequence, companies need to adapt their material management taking into account inbound flows of used products. Corresponding inventory control models have been proposed in literature. In this paper we address the issue of planning stability in a product recovery context. To this end, we consider rolling horizon planning for a stock point facing stochastic demand and product returns. We analyze the impact of the return flow on planning stability and compare the system behaviour with a traditional production environment. We show that structural results derived for traditional inventory models remain valid in a product recovery context. Moreover we discuss counterintuitive effects resulting from interaction between planning stability and stock levels.
\end{abstract}

Zusammenfassung. In den letzten Jahren besteht aufgrund gesetzlicher Bestimmungen und gestiegenem Umweltbewußtsein in der Bevölkerung zunehmend die Tendenz, daß Unternehmen ihre Produkte nach deren Gebrauch vom Kunden zurücknehmen. Die Produktionsplanung und -steuerung der Unternehmen muß diesen Produktrückflüssen angepaßt werden. In der Literatur sind für verschiedene kreislaufwirtschaftliche Probleme optimale Lagerhaltungspolitiken abgeleitet worden. Dieser Beitrag beschäftigt sich mit der Planungsstabilität in einem kreislaufwirtschaftlichen Basismodell, wo alle zurückkommenden Produkte aufgearbeitet werden müssen. Insbesondere wird der Einfluß der Produktrückflüsse auf die Stabilität untersucht und ein Vergleich mit der Stabilität eines traditionellen Lagerhaltungsmodells durchgeführt. Es wird aufgezeigt, daß beide Modelle im wesentlichen dieselben strukturellen Eigenschaften besitzen. 
Key words: Reverse logistics - Nervousness - Rolling horizon planning - Inventory control rules

Schlüsselwörter: Logistik für Kreislaufwirtschaftsprozesse - Planungsnervosität - Rollierende Planung - Lagerhaltungspolitiken

\section{Introduction}

Enhanced producer responsibility is one of the consequences of growing environmental concern in recent years. Legislation and environmentally conscious customers increasingly force manufacturers to take back their products after use. As alternatives are sought to waste disposal and incineration, efforts are made to reintegrate used products into industrial production processes. Besides mandatory recovery quotas and a 'green' image there are also economic drivers for recovering used products. Reuse of products may lead to savings in material, manufacturing and disposal costs. Examples from practice include reusable packages, electronic scrap recycling, and car part remanufacturing. The management of the material flow concerned with the recovery of returned products, which is opposite to the usual supply chain flow, is considered in the recently emerged field of 'reverse logistics' [2].

One of the issues in this area is production and inventory management. The major task in this field is to develop appropriate planning and control methods in order to integrate the return flow of used products into the producers' material management. Difficulties arise because of the considerable uncertainties in timing, quantity, and quality of the return flow which is often hard to influence by the producer. One of the consequences of this lack of control is an increase of the stock levels in the entire system.

Several inventory models and control policies for product recovery systems have been proposed in literature. In an early paper Simpson [15] considers the trade-off between material savings due to reuse versus additional inventory carrying costs and proves optimality of a three parameter policy to control order, repair, and disposal for the case without fixed costs and leadtimes. Inderfurth [9] shows this policy to remain optimal in the case of fixed and identical leadtimes for repair and procurement. Fleischmann and Kuik [3] consider another variant of this model excluding disposal and prove average cost optimality of an $(s, S)$-policy. In continuous review models $(s, Q)$-type-policies have been discussed, see, e.g., Heyman [7], Muckstadt and Isaac [14], and Van der Laan et al. [17,18]. A more detailed literature review is given by Fleischmann et al. [2].

In most practical situations the stochastic environment is taken into account by a rolling horizon planning framework. Then, the replenishment decisions, i.e. production and remanufacturing orders, are determined on the basis of a quasideterministic modeling together with a periodic updating of all relevant parameters and subsequent replanning activities. Rolling horizon planning leads to replanning activities caused by permanent processing of new information in successive planning cycles. Consequently, formerly fixed order decisions are replanned in later 
periods. This discontinuity in order releases is known as the nervousness syndrome (see [19]).

In the recovery context the question arises as to how system nervousness is influenced by the integration of product returns into the material management. Since uncertainty in the planning process rises by incorporating product returns one may expect planning stability to decrease, similar to the impact of increasing demand uncertainty in traditional inventory systems (see, e.g., [1], [4]). In this paper we address this intuitive relation more systematically.

Lack of planning stability often generates a considerable amount of short- and medium-term adjustment efforts as well as a general loss of planning confidence. In particular, in a multi-stage production environment nervousness on the top level is propagating throughout the system. Since in many cases the consequences of replanning activities cannot be valued in terms of costs, we treat planning stability as an additional attribute for assessing inventory control systems, similar to the attribute of customer service. Therefore, we have to define a general quantitative stability measure. A systematic development of nervousness measures is given in, e.g., [1], [8], and [11]. We can distinguish short-term planning instability, which measures nervousness with respect to only the first period's order decision, and long-term planning instability, where all replenishment decisions over the entire planning horizon are compared. Moreover, it is common to differentiate between quantity adjustments, which is known as quantity-oriented instability, and changes in order setups, i.e. either cancelling a previously planned order or introducing an additional setup in a new planning cycle. The latter kind of nervousness is called setup-oriented instability. In this paper, we examine setup stability, because in many practical situations it is the fixed effort caused by replanning an order decision that hinders the execution of a planning process, independent of the specific quantity adjustment.

Up to now the issue of planning stability has mainly been treated in 'traditional' production and inventory management systems where control rules such as $(R, S)$-, $(s, n Q)$ - or $(s, S)$ - policies are used. In [8] the performance of the $(s, S)$ - and $(s, n Q)$ - policy for uniformly and exponentially distributed demand with respect to a short-term setup-oriented planning stability is analyzed. In [1] short-term setup- as well as quantity-oriented stability of orders for more general demand distributions are examined for $(s, S)$-, $(s, n Q)$ - and $(R, S)$ - policies. In [4] the long-term setuporiented stability performance of an $(s, S)$ - policy is analyzed for general demand distributions. Finally, [5] deals with setup stability for a simple modified order-upto-level $S$-policy in a product recovery system.

In this paper we analyze the impact of product recovery on stability in a basic model. Insights from this model, which allows for a detailed analysis, may provide a basis for addressing more complex situations in future research. The most basic characteristic distinguishing inventory management in a reuse context from traditional settings is the existence of an exogenous item in-flow. This is also the common core of the recovery models mentioned above. Given this key element of a recovery environment we explicitly address the influence of return flows on planning stability in this paper. We consider a model of one inventory point where serviceable items are stored. For this model, which was introduced by Fleischmann 
and Kuik, we provide an analysis of setup stability of the optimal policy. In Inderfurth and Jensen [10] an MRP-framework for this model can be found. Within this so-called MRRP (Materials Requirements and Recovery Planning) approach the replenishment decisions are not only planned on basis of forecasted customer demand, but also take into account the projected returns of used products. Our analysis is closely related to that of traditional stability analysis, in particular to the work of Heisig [4]. We show how these results change by integrating a product return flow.

The remainder of the paper is organized as follows. In Section 2 we introduce and formalize the examined recovery management problem. In Section 3 a measure for nervousness under rolling planning conditions is given. In Section 4 we derive analytical expressions for the setup stability of production and recovery decisions. In Section 5 we present a numerical example showing the influence of several system parameters. In particular, we study the impact of the return flow on stability. Section 6 summarizes our results and gives directions for further research.

\section{The product recovery system}

In this section we introduce the product recovery system considered and give a formal definition of the corresponding mathematical model and notation. As discussed in the introduction we address the impact of recoverable product returns on planning stability. To this end, we consider a traditional single item inventory system with an additional in-flow of used items. Returned items undergo a recovery process (which we denote by remanufacturing in the sequel, although other processes such as repair or cleaning and testing are also included) and can then be used as an alternative to new production to satisfy demand. Figure 1 gives a graphical representation of this system. As examples one may think of reusable packages, such as crates and containers, or spare parts disassembled from used equipment, such as in the electronics and automotive industries [16]. Moreover, as pointed out before this situation is at the core of most other, more complex product recovery systems including, e.g., additional stock points or control options. Typically, recovery of used products is significantly cheaper than regular production. Therefore, the major challenge in this context concerns the tradeoff between the savings potential and the uncertain availability of the recovery source. To model the above system we assume that demand and returns are given by independent stochastic processes.

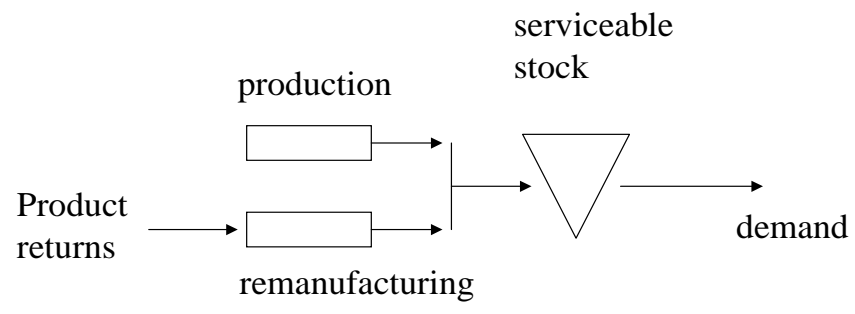

Fig. 1. A basic product recovery system 
Orders for remanufacturing and production are placed on a periodic review basis. Any unsatisfied demand is backordered. For ease of notation we assume that all orders are delivered instantaneously (leadtimes can be incorporated in the standard way by appropriately redefining the inventory position, under the condition that the remanufacturing leadtime does not exceed the manufacturing leadtime; see [3]). As indicated above, remanufacturing is implemented as a push process, i.e. at the beginning of each period all products returned during the previous period are remanufactured. Production orders are the decision variables to control the system. We use the following notation. Let

$D_{t}=$ demand in period $t$

$R_{t}=$ returns in period $t$

$N_{t}=D_{t}-R_{t}$, net demand in period $t$,

where $\left(N_{t}\right)_{t \in \mathbb{N}}$ is an i.i.d. sequence of random variables distributed as a random variable $N$ with continuous distribution function $\Phi$, density $\phi$, and expected value $\mathbb{E}[N]$. Since our model does not include a disposal option we assume that $\mathbb{E}[N]>0$ since the inventory level would increase to infinity otherwise. Under these circumstances, Fleischmann and Kuik [3] have shown average cost optimality of a stationary $(s, S)$-policy for production under a fixed plus convex cost structure. Therefore, we assume in the sequel that production orders are placed according to a stationary $(s, S)$-rule. Let

$O_{t} \quad=\quad$ remanufacturing order size in period $t$;

$Z_{t} \quad=\quad$ net stock at the beginning of period $t$ after remanufacturing, before production;

$Q_{t}=$ production order size in period $t$;

$Y_{t}=$ net stock at the beginning of period $t$ after remanufacturing and production;

$s \quad=\quad$ production reorder level;

$S=$ production order upto level.

Then the system dynamics are described by

$O_{t}=R_{t-1}$

$Z_{t} \quad=Y_{t-1}-N_{t-1}$

$Q_{t}= \begin{cases}0 & \text { if } Z_{t}>s \\ S-Z_{t} & \text { else }\end{cases}$

$Y_{t}=Z_{t}+Q_{t}$.

It is worth noting that these relations are the same as in a traditional $(s, S)$ inventory system up to the difference that the (net) demand may be negative. In particular, $\left(Y_{t}\right)_{t \in \mathbb{N}}$ forms a discrete time Markov process on $[s, \infty)$. Note that in contrast with traditional inventory systems the net inventory is unbounded from above, as a consequence of the stochastic return flow. More specifically, $\left(Y_{t}\right)_{t \in \mathbb{N}}$ is a random walk, which is ergodic due to the condition $\mathbb{E}[N]>0$ (see [3] for a formal proof). Therefore, $\left(Y_{t}\right)$ admits a limiting stationary distribution, which we denote by $F_{Y}$. Furthermore, let $Y$ denote a corresponding random variable. 
As discussed before, we address planning stability by analyzing the performance of the above system in a rolling planning horizon setting, such as in a typical MRP environment. We refer to Inderfurth and Jensen [10] for a general discussion of an MRP-framework including product recovery. Moreover, we remark that Heisig [5] analyzed planning stability in the above system for the special case of a simple order-upto- $S$ policy for production orders. In what follows we extend this approach to the more general case of an $(s, S)$-policy.

Hence, let us assume that at the beginning of each period a planning cycle of $T+1$ periods is started, where $T$ is some fixed planning horizon. That is, remanufacturing and production orders are planned for the current plus the next $T$ periods. Orders for the current period are released immediately, whereas planned orders for future periods are only preliminary and may be updated in later periods. Conversely, in each period the orders for the current plus the next $T-1$ periods are updates of previous plans. We denote by $\hat{Q}_{t}^{i}\left(\hat{O}_{t}^{i}\right)$ the production (remanufacturing) order size in period $t$ as planned in period $i$. Analogously, $\hat{Z}_{t}^{i}\left(\hat{Y}_{t}^{i}\right)$ denotes the net stock at the beginning of period $t$ before (after) production as planned in period $i$. Finally, let $\hat{N}$ denote the projected net demand per period and $\hat{D}$ and $\hat{R}$ the projected (gross) demand and returns per period, respectively. Note that these projections need not necessarily coincide with the corresponding expected values. In particular, setting $\hat{R}=0$ is a common choice alternative to $\hat{R}=\mathbb{E}\left[R_{t}\right]$. These two cases are referred to as reactive and proactive recovery planning, respectively [10].

To sum up, at the beginning of each period $t$ a sequence of planned production orders $\left(Q_{t}, \hat{Q}_{t+1}^{t}, \hat{Q}_{t+2}^{t}, \ldots, \hat{Q}_{t+T}^{t}\right)$ and remanufacturing orders $\left(O_{t}, \hat{O}_{t+1}^{t}, \hat{O}_{t+2}^{t}, \ldots\right.$, $\hat{O}_{t+T}^{t}$ ) is generated (see also Figure 2). The system dynamics are analogous to the above except that demand and returns are replaced by their projected values. Hence,

$\hat{O}_{t+i}^{t}=\hat{R}$

$\hat{Z}_{t+i}^{t}=\hat{Y}_{t+i-1}^{t}-\hat{N}$

$\hat{Q}_{t+i}^{t}= \begin{cases}0 & \text { if } \hat{Z}_{t+i}^{t}>s \\ S-\hat{Z}_{t+i}^{t} & \text { else }\end{cases}$

$\hat{Y}_{t+i}^{t}=\hat{Z}_{t+i}^{t}+\hat{Q}_{t+i}^{t}$

for $i=1, \ldots, T$ (where we define $\hat{Z}_{t}^{t}=Z_{t}$ and analogously for $Y_{t}, Q_{t}$, and $O_{t}$ ). Planning stability then characterizes the variation of $\hat{Q}_{t}^{i}$ and $\hat{O}_{t}^{i}$ as a function of $i$, in other words, the difference between subsequent planning updates. We define this notion more rigorously in the next section. Here, we conclude by noting that successive planned orders $\hat{Q}_{t+1}^{t}$ and $Q_{t+1}\left(\hat{O}_{t+1}^{t}\right.$ and $\left.O_{t+1}\right)$ may differ whenever $N_{t}\left(R_{t}\right)$ deviates from its projected value $\hat{N}(\hat{R})$.

\section{Measuring planning stability}

In Figure 2 the above relations are summarized for two successive planning cycles. Here, $q_{j}^{t}\left(\hat{q}_{j}^{t}\right)$ may represent both the (planned) production or remanufacturing decision in period $j$ for planning cycle $t$. The production/remanufacturing order $q_{t}$ is released immediately while future decisions are only preliminary. After period 


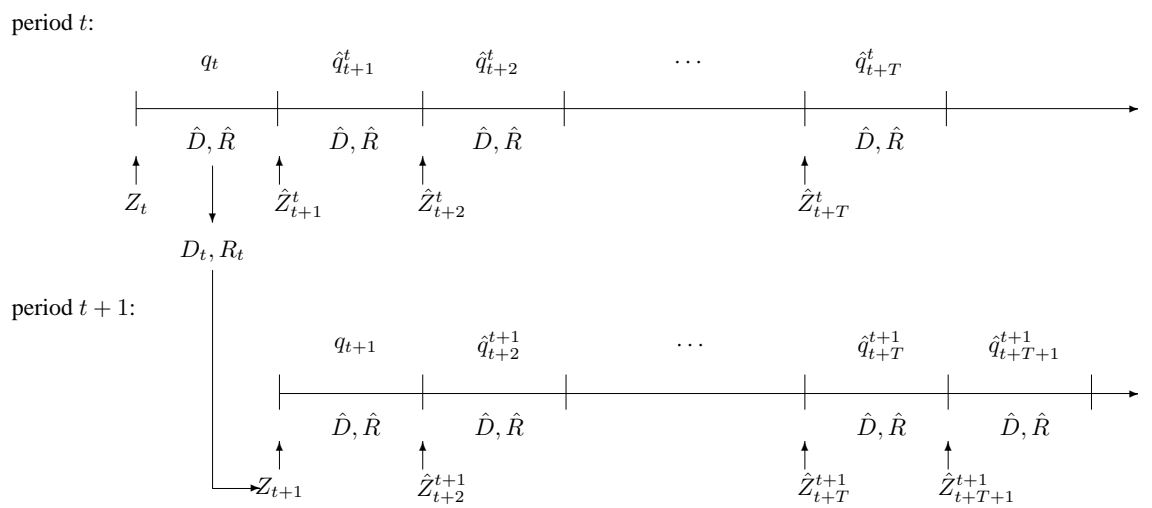

Fig. 2. Decisions and influences in a product recovery system for two consecutive planning cycles

$t$ has passed, a subsequent planning cycle $t+1$ starts. A change of the planned replenishment may only occur if actual net demand $D_{t}-R_{t}$ differs from its forecast $\hat{D}-\hat{R}$. Then, the actual inventory position $Z_{t+1}$ deviates from its planned value $\hat{Z}_{t+1}^{t}$ as calculated in the previous planning cycle. Updating the starting inventory $Z_{t+1}$ and applying the $(s, S)$-policy generates an adjusted sequence of order releases as depicted in Figure 2.

In the sequel, we briefly summarize concepts from literature for measuring planning stability (see e.g. [1], [4], [8] [11], [12], and [13]). In consecutive planning cycles each period is scheduled $T$ times allowing for a replanning of former replenishment decisions. This is related with nervousness in the planning process. Changes may be caused by additional planning information as well as by realizations of the stochastic variables of the system. Nervousness, in general, arises when a formerly fixed order decision for a certain period is replanned in a later planning cycle, i.e. if we find for any $j$ and $v$ :

$$
q_{j}^{t} \neq q_{j}^{t+v} \quad \text { for } \quad j \geq t \geq v>0 .
$$

This kind of instability can be characterized as planned orders nervousness. Since we consider a stationary inventory policy (i.e. control parameters change neither across periods nor across planning cycles) it is sufficient to determine the nervousness for two arbitrary successive planning cycles as shown in Figure 2, i.e. setting $v=1$ in (1). Then, a measure of nervousness $\nu$ can be defined as (see e.g. [4])

$$
\nu=\frac{\sum_{i=1}^{T}\left|\delta\left(q_{t+i}^{t}\right)-\delta\left(q_{t+i}^{t+1}\right)\right|}{T},
$$

where $\delta(q)=0$, for $q=0$, and $\delta(q)=1$ otherwise. Since the setup decisions in cycle $t$ and $t+1$ depend on the stochastic stock level and (net) demand, $\nu$ is a random variable. The measure of setup stability is now given by

$$
\pi=\mathbb{E}[1-\nu] .
$$


The formalized measure of nervousness $\nu$ in (2) is characterized by relating the number of periods with changed setups to the total number of periods that can be compared. Thus $\nu$ and $\pi$ are restricted to the value space $[0,1]$ where the value $\pi=0$ refers to maximum nervousness, while extreme setup planning stability is characterized by $\pi=1$. Since this measure takes into account all comparable order decisions over the entire stability horizon it is called long-term planning stability. As a special case, short-term planning stability is obtained for $T=1$ (see e.g. [8]). The measure in (2) can be applied to determine the setup stability on both stages in our product recovery system. With $q_{t+i}^{t}=Q_{t+i}^{t}$ in (2) we can measure stability on production stage, and by setting $q_{t+i}^{t}=R_{t+i}^{t}$ we get setup stability for the remanufacturing stage.

In a steady-state situation considering two successive planning cycles the initial inventory $Z_{t}$ at the beginning of cycle $t$, the planned orders $\hat{q}_{t+i}^{t}(i=1, \ldots, T)$, and (with an additional stochastic influence from $D_{t}-R_{t}$ ) the replenishment sequence in planning cycle $t+1\left(q_{t+1} ; \hat{q}_{t+i}^{t+1}, i=2, \ldots, T\right)$ are random variables. Stability can be interpreted as the average probability that the setup decisions do not change from planning cycle $t$ to cycle $t+1$, i.e.

$$
\begin{aligned}
\pi= & \frac{1}{T}\left[\mathbb{P}\left\{\delta\left(\hat{q}_{t+1}^{t}\right)=\delta\left(q_{t+1}\right)\right\}+\mathbb{P}\left\{\delta\left(\hat{q}_{t+2}^{t}\right)=\delta\left(\hat{q}_{t+2}^{t+1}\right)\right\}+\right. \\
& \left.\ldots+\mathbb{P}\left\{\delta\left(\hat{q}_{t+T}^{t}\right)=\delta\left(\hat{q}_{t+T}^{t+1}\right)\right\}\right] .
\end{aligned}
$$

In order to calculate setup stability for production and remanufacturing orders as indicated in (4) we have to determine the sequence of these setups. This is done in the next section. Moreover, we derive a general expression for $\pi$.

\section{Stationary stability analysis}

In this section we analyze the planning stability of the product recovery model introduced in Section 2. As discussed in the previous section, expected planning stability in steady state is determined by the planned order decisions generated in two consecutive planning cycles. In order to evaluate the stability measure $\pi$ introduced in the previous section we analyze the structure of the planned order sequences $\left(Q_{t}, \hat{Q}_{t+1}^{t}, \hat{Q}_{t+2}^{t}, \ldots, \hat{Q}_{t+T}^{t}\right)$ and $\left(O_{t}, \hat{O}_{t+1}^{t}, \hat{O}_{t+2}^{t}, \ldots, \hat{O}_{t+T}^{t}\right)$ in more detail.

We first address the production orders. Consider the planning cycles starting in periods $t$ and $t+1$, respectively. To compute $\pi$ we need to take into account the planned orders $\hat{Q}_{i}^{j}$ for $j=t, t+1$ and $i=t+1, \ldots, t+T$ (see (2)). From the transition rules as discussed in Section 2 we have that $\hat{Q}_{t+i}^{t}$ for $i \geq 1$ equals 0 whenever the following condition holds

$$
\hat{Z}_{t+i}^{t}>s \Longleftrightarrow \hat{Y}_{t+i-1}^{t}-\hat{N}>s \Longleftrightarrow \hat{Z}_{t+i-1}^{t}-\hat{N}+\hat{Q}_{t+i-1}^{t}>s .
$$

Hence, we obtain by induction that the smallest $\tau \geq 1$ for which $\hat{Q}_{t+\tau}^{t}>0$ satisfies $(\tau-1) \hat{N} \leq Y_{t}-s<\tau \hat{N}$. Therefore, the first order after period $t$ is planned for 
period $t+\tau$ where

$$
\tau=\tau\left(Y_{t}\right)=\left\lceil\frac{Y_{t}-s}{\hat{N}}\right\rceil
$$

In the same way we find that the next order is planned for period $t+\tau+l$ where

$$
l=\left\lceil\frac{\hat{Y}_{t+\tau}^{t}-s}{\hat{N}}\right\rceil=\left\lceil\frac{S-s}{\hat{N}}\right\rceil,
$$

where $\hat{Y}_{t+\tau}^{t}=S$ follows from the definition of $\tau$. Note that $l$ characterizes the expected coverage of the minimum order size $S-s$ or, in other words, the expected time between ordering. Subsequently, the same step is repeated until the end of the planning horizon is reached. Summing up, we find that in the planning cycle starting in an arbitrary period $t$ production orders after $t$ are planned for the periods $t+\tau+k \cdot l$ for $k \in \mathbb{N}$ until the end of the planning horizon. If $\tau>T$ then no order is planned during the entire planning horizon.

Applying this structural result to the planning cycle starting in period $t+1$ yields that the first order after $t+1$ is planned in period $t+1+\left\lceil\left(Y_{t+1}-s\right) / \hat{N}\right\rceil=$ $t+1+\left\lceil\left(Y_{t}-N_{t}-s+Q_{t+1}\right) / \hat{N}\right\rceil$. In addition, we now have to take into account the decision for period $t+1$, i.e. $Q_{t+1}$. We have that $Q_{t+1}>0 \Leftrightarrow Y_{t}-N_{t}-s \leq 0$. Combining both results yields that the smallest $\theta \geq 1$ for which $\hat{Q}_{t+\theta}^{t+1}>0$ is given by

$$
\theta=\theta\left(Y_{t}, N_{t}\right)=1+\max \left\{0,\left\lceil\frac{Y_{t}-N_{t}-s}{\hat{N}}\right\rceil\right\} .
$$

Thereafter, orders are again planned every $l$ periods until the end of the planning horizon. Hence, in this planning cycle orders are planned for periods $t+\theta+k \cdot l$ for $k \in \mathbb{I N}$.

The above considerations show that the planned order sequences generated in each planning cycle have a fairly simple, regular structure. Planning updates in subsequent planning cycles result in a shift of (stochastic) length $\tau-\theta$ of the entire planned order sequence. We point out that the same structure is found for traditional $(s, S)$ production systems without product recovery [4]. However, the range of possible values for $\tau$ and $\theta$ are different in both cases. In traditional production systems we have that $\tau \leq l$ and $\theta \leq \tau+1$. Both relations do not hold in our product recovery system since the net demand in a given period may be negative and the net stock may exceed $S$. Moreover, the probability distributions of $\tau$ and $\theta$ do, of course, depend on the product returns. We investigate this dependence in more detail in the next section.

Furthermore, it is worth noting that for $S-s<\hat{N}$ we have $l=1$, i.e. an order is planned for every period after the first setup and the above formulas reduce to the results for a base stock system [5].

Finally, note that for $S-s>>\hat{N}$ we may approximate $\tau$ and $\theta$ by

$$
\tau(Y) \approx \frac{Y-s}{\hat{N}} \quad \text { and } \quad \theta(Y, N) \approx 1+\frac{Y-N-s}{\hat{N}} .
$$


Hence, we have in this case that

$$
\mathbb{E}\left[(\tau(Y)-\theta(Y, N))^{2}\right] \approx \mathbb{E}\left[\frac{(\hat{N}-N)^{2}}{\hat{N}^{2}}\right]=\frac{\operatorname{Var}[N]}{\mathbb{E}[N]^{2}}
$$

Hence, the expected squared length of the shift of the order sequence during a planning update is approximately equal to the squared coefficient of variation of the net demand per period. Note that this expression is increasing in both the expected value and the variance of the returns per period. This gives a first indication of the impact of the product returns on the planning updates.

We can now use the above results to evaluate the stability measure $\pi$ introduced in the previous section. To this end, we introduce some additional notation as follows. Let $V_{t}:=Y_{t}-s$ for all $t$. Then $V_{t} \in[0, \infty)$ for all $t$. Moreover, recall from Section 2 that $Y_{t}$ admits a stationary limiting distribution. Hence, the same holds for $V_{t}$ and we denote the limiting distribution by $F_{V}$. Moreover, let $V$ be a corresponding random variable.

Furthermore, for $\tau, \theta=1, \ldots T+1$ we define the stability quotient $\beta_{\theta}^{\tau}$ as the number of periods with identical setup decisions in two subsequent planning cycles with first setups in periods $\tau$ and $\theta$ divided by the length of the planning horizon $T$. Note that $\beta_{\theta}^{\tau}$ equals the planning stability $\pi$ for fixed values of $\tau$ and $\theta$ (see (2)). While there is no simple closed form expression for the stability quotients these values can easily be computed by enumeration. For $\tau>T+1$ we set $\beta_{\theta}^{\tau}=\beta_{\theta}^{T+1}$ and analogously for $\theta>T+1$. Integrating over the random variables $\tau=\tau(Y)$ and $\theta=\theta(Y, N)$ we can now derive the following general expression for the expected planning stability in steady state.

$$
\begin{aligned}
\pi= & \int_{s}^{\infty} \int_{-\infty}^{\infty} \beta_{\theta(y, n)}^{\tau(y)} d \Phi(n) d F_{Y}(y) \\
= & \sum_{\tau=1}^{\infty} \int_{(s+(\tau-1) \hat{N}, s+\tau \hat{N}]}\left[\sum_{\theta=1}^{\infty} \int_{[y-s-(\theta-1) \hat{N}, y-s-(\theta-2) \hat{N})} \beta_{\theta}^{\tau} d \Phi(n)\right. \\
& \left.+\int_{[y-s+\hat{N}, \infty)} \beta_{1}^{\tau} d \Phi(n)\right] d F_{Y}(y) \\
= & \sum_{\tau=1}^{\infty} \int_{(s+(\tau-1) \hat{N}, s+\tau \hat{N}]}\left[\sum_{\theta=1}^{\infty} \beta_{\theta}^{\tau} \int_{y-s-(\theta-1) \hat{N}}^{y-s-(\theta-2) \hat{N}} \phi(n) d n\right. \\
& \left.+\beta_{1}^{\tau} \int_{y-s+\hat{N}}^{\infty} \phi(n) d n\right] d F_{Y}(y) \\
= & \sum_{\tau=1}^{\infty} \int_{((\tau-1) \hat{N}, \tau \hat{N}]}^{\infty}\left[\sum_{\theta=1}^{\infty} \beta_{\theta}^{\tau} \int_{v-(\theta-1) \hat{N}}^{v-(\theta-2) \hat{N}} \phi(n) d n\right. \\
& \left.+\beta_{1}^{\tau} \int_{v+\hat{N}}^{\infty} \phi(n) d n\right] d F_{V}(v)
\end{aligned}
$$


Note that $\pi$ is independent of the reorder level $s$ just as in traditional production systems. Therefore, $s$ can be chosen according to cost and service considerations without influencing planning stability. In the next section we compute the value of $\pi$ in an example and analyze its dependence on the system parameters.

We conclude this section by briefly considering the planning stability of the remanufacturing decisions. Since we consider a push-strategy where all items available are remanufactured at the beginning of each period and since $R_{t}$ is stationary

we have that $\hat{O}_{t+i}^{t}=\hat{R}$ for all $t$ and all $i \geq 1$. Hence, two consecutive planning cycles can only differ with respect to the remanufacturing order in the first period. This is analogous with a base-stock policy in a traditional production system where an order is placed in each period except for the case of no demand. The specific value of the remanufacturing oriented planning stability in our model depends on the choice of $\hat{R}$. For $\hat{R}=0$ (reactive strategy, compare Section 2) the stability measure equals $\left(\mathbb{P}\left\{R_{t}=0\right\}+T-1\right) / T=(T-1) / T$. In all other cases we obtain maximum stability $\left(\mathbb{P}\left\{R_{t}>0\right\}+T-1\right) / T=1$.

\section{Numerical example}

In this section we illustrate the dependence of planning stability on the different system parameters in a numerical example. In order to obtain easily tractable expressions we assume both demand and returns to be exponentially distributed. For traditional inventory systems the specific form of the demand distribution has been found to have a rather limited effect on the properties of the stability function (see, e.g., $[1,4]$ ). While the overall level of planning stability tends to decrease for a growing coefficient of variation, qualitative properties remain largely unchanged. One may expect a similar robustness of the stability function in our product recovery system.

In Section 5.1 below we derive an analytic expression of the aforementioned stability measure for the case of exponentially distributed demand and returns. In Section 5.2 we evaluate this measure for different parameter settings. Finally, Section 5.3 focuses on the impact of the return volume specifically.

\subsection{The stability function for exponentially distributed demand and returns}

For exponentially distributed demand and returns the probability density of net demand $N$ is of the form

$$
\phi(n)= \begin{cases}\frac{\mu \lambda}{\mu+\lambda} e^{-\lambda n} & \text { for } \quad n \geq 0 \\ \frac{\mu \lambda}{\mu+\lambda} e^{\mu n} & \text { for } \quad n<0\end{cases}
$$

where $\mathbb{E}[D]=\frac{1}{\lambda}$, and $\mathbb{E}[R]=\frac{1}{\mu}$ are the expected demand and returns, respectively. As discussed in Section 2, we assume that $\mu>\lambda$. Defining $Q:=S-s$ as the minimum order lot size the stationary distribution of $V$ can now be written as (see Appendix A) 


$$
F_{V}(v)=\int_{-\infty}^{v} f_{V}(x) d x+1_{v \geq Q} p_{Q},
$$

with $f_{V}(v)= \begin{cases}0 & \text { for } v<0 \\ c e^{-(\mu-\lambda) v} & \text { for } v \geq 0\end{cases}$

$$
\text { and } p_{Q}=\frac{\mu-\lambda}{\mu\left(2-e^{-\lambda Q}\right)}
$$

where $\quad c:=p_{Q}\left[\lambda+\mu\left(1-e^{-\lambda Q}\right)\right]$.

Using these expressions, the stability measure $\pi$ is given as follows (see Appendix B). We distinguish two cases.

Case A: For $l \leq T$ we have

$$
\begin{aligned}
\pi=\frac{c_{\hat{N}}}{\mu+\lambda} & +\frac{p_{Q}}{\mu+\lambda}\left[\beta_{1}^{l} \mu e^{-\lambda Q}+\sum_{\theta=2}^{l} \beta_{\theta}^{l} \mu c_{\lambda} e^{-\lambda(Q-(\theta-1) \hat{N})}\right. \\
& +\beta_{l+1}^{l}\left[\mu+\lambda-\mu\left(1-c_{\lambda}\right) e^{-\lambda(Q-l \hat{N})}\right. \\
& \left.\left.-\lambda e^{\mu(Q-l \hat{N})}\right]+\sum_{\theta=l+2}^{T+1} \beta_{\theta}^{l} \lambda c_{\mu} e^{\mu(Q-(\theta-1) \hat{N})}+\beta_{T+1}^{l} \lambda c_{T} e^{\mu Q}\right]
\end{aligned}
$$

Case B: For $l>T$ we have

$$
\begin{gathered}
\pi=\frac{c_{\hat{N}}}{\mu+\lambda}+\frac{p_{Q}}{\mu+\lambda}\left[\beta_{1}^{T+1} \mu e^{-\lambda Q}+\sum_{\theta=2}^{T+1} \beta_{\theta}^{T+1} \mu c_{\lambda} e^{-\lambda(Q-(\theta-1) \hat{N})}\right. \\
\left.+\beta_{T+1}^{T+1}\left(\mu+\lambda-\mu e^{-\lambda(Q-T \hat{N})}\right)\right]
\end{gathered}
$$

where

$$
\begin{aligned}
c_{\hat{N}}:=c\left\{\sum_{\tau=1}^{T}\right. & {\left[\beta_{1}^{\tau} c_{\mu} e^{-\mu \tau \hat{N}}+\sum_{\theta=2}^{\tau} \beta_{\theta}^{\tau} c_{\mu} c_{\lambda} e^{-(\mu \tau-\lambda(\theta-1)) \hat{N}}\right.} \\
& +\beta_{\tau+1}^{\tau} \frac{2}{\mu-\lambda}\left(\lambda c_{\mu}\left(1-c_{\lambda}\right)-\mu c_{\lambda}\right) e^{-(\mu-\lambda) \tau \hat{N}} \\
& \left.+\sum_{\theta=\tau+2}^{T+1} \beta_{\theta}^{\tau} c_{\mu} c_{\lambda} e^{-(\mu(\theta-1)-\lambda \tau) \hat{N}}+\beta_{T+1}^{\tau} c_{T} c_{\lambda} e^{\lambda \tau \hat{N}}\right] \\
& \left.+\beta_{1}^{T+1} c_{T}+\sum_{\theta=2}^{T+1} \beta_{\theta}^{T+1} c_{T} c_{\lambda} e^{\lambda(\theta-1) \hat{N}}+\beta_{T+1}^{T+1} \frac{2 \lambda}{\mu-\lambda} e^{-(\mu-\lambda) T \hat{N}}\right\}
\end{aligned}
$$

and $c_{\mu}:=\left(e^{\mu \hat{N}}-1\right), c_{\lambda}:=\left(1-e^{-\lambda \hat{N}}\right)$, and $c_{T}:=e^{-\mu T \hat{N}}$.

The distinction between $T \leq l$ and $T>l$ is necessary since $F_{V}$ has a positive probability mass at $v=Q$. In the first case $Q$ lies in the interval $\tau=l$ whereas in 
the second case $Q$ belongs to $\tau=T+1$. Thus the second term in (11) differs from the second one in (12).

Observe that planning stability only depends on the lot size parameter $Q=S-$ $s$, the length of the stability horizon $T+1$, and the demand and return expectations $\bar{D}, \bar{R}$ and forecasts $\hat{D}, \hat{R}$ in this case. We recall that the forecasts may, in general, deviate from the corresponding expectations. However, since our main goal is to analyze the impact of product returns on stability, we now assume both values to coincide, in order to avoid overlapping effects. Hence, in the remainder of this section we set $\bar{D}=\hat{D}, \bar{R}=\hat{R}$, and consequently $\mathbb{E}[N]=\hat{N}$.

\subsection{Numerical evaluation of the stability measure}

We now illustrate the influence of $Q, T$ and $\hat{D}$ as well as $\hat{R}$ on the planning stability in a number of numerical examples. We use mainly the same parameter setting as in [4], except for a larger (net) demand expectation. The parameter values are summarized in Table 1 . We use $T=1$ to represent short-term considerations, and $T=50$ for an 'infinite' planning horizon.

First, we examine the impact of $Q$ and $T$ on stability, and compare the results for our recovery model and for traditional inventory models. To this end, we consider a conventional $(s, S)$-inventory model with exponentially distributed demand and a demand intensity that is equal to the expected net demand in the recovery model, i.e. $\hat{D}=10$. Figures 3 and 4 show the shape of the stability function for both models.

We observe that the results are fairly similar for both cases. Moreover, the stability level in the recovery model tends to be lower than in the traditional model in most cases. For $Q \rightarrow \infty$ stability tends to $100 \%$ in both models, because almost no setup takes place. While stability also reaches $100 \%$ for $Q \rightarrow 0$ in the traditional model we observe significantly smaller values of $\pi$ in the recovery model in the case of a small planning horizon. The shorter the planning horizon the lower the resulting stability level. This different behaviour of the stability function can be explained as follows. For $Q \leq \hat{D}$ a setup occurs in almost each period in the traditional model. Only if the realized demand is very small, there is no setup in the first period of the second planning cycle (see [4]). In particular, a setup is planned for each period in the first planning cycle. In contrast, there may be some periods without planned setups in cycle $t$ (as well as in cycle $t+1$ ) in the recovery model, due to the unbounded initial stock. Therefore, the level of stability is lower, in particular for small planning horizons.

Furthermore we find for both models that the shape of the stability function is continuous for $l>T$, whereas it is discontinuous for $l \leq T$ at the points

Table 1. Parameter settings Figure 4

\begin{tabular}{l|c|c}
\hline$\hat{D}$ & $\hat{R}$ & $\mathrm{~T}$ \\
\hline 20 & 10 & $1,8,50$ \\
\hline
\end{tabular}




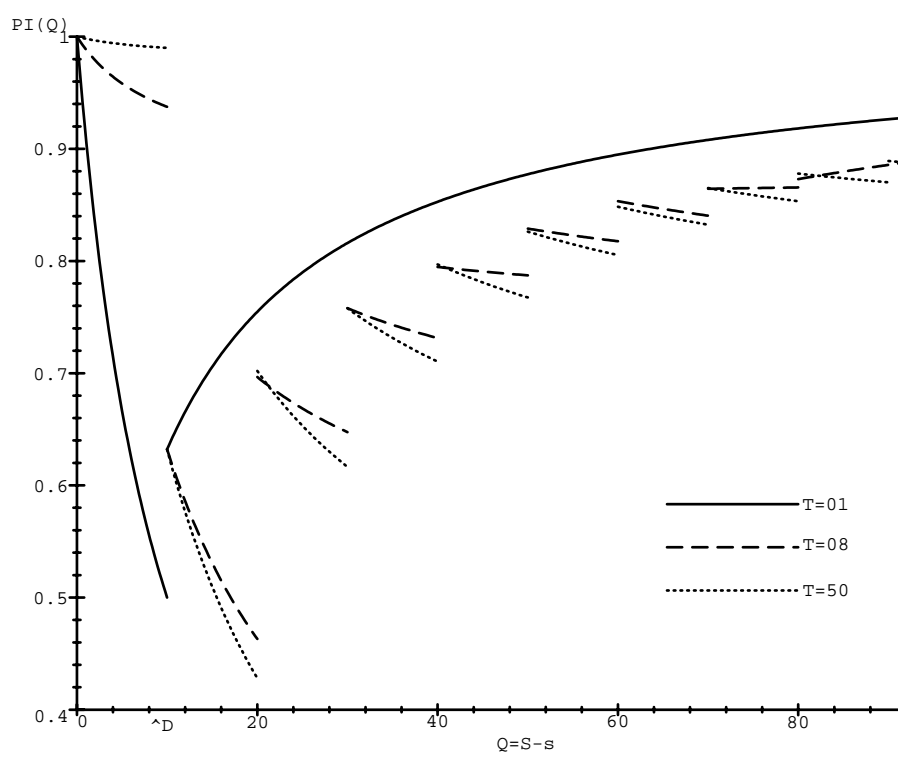

Fig. 3. Stability function in traditional model (for different planning horizons)

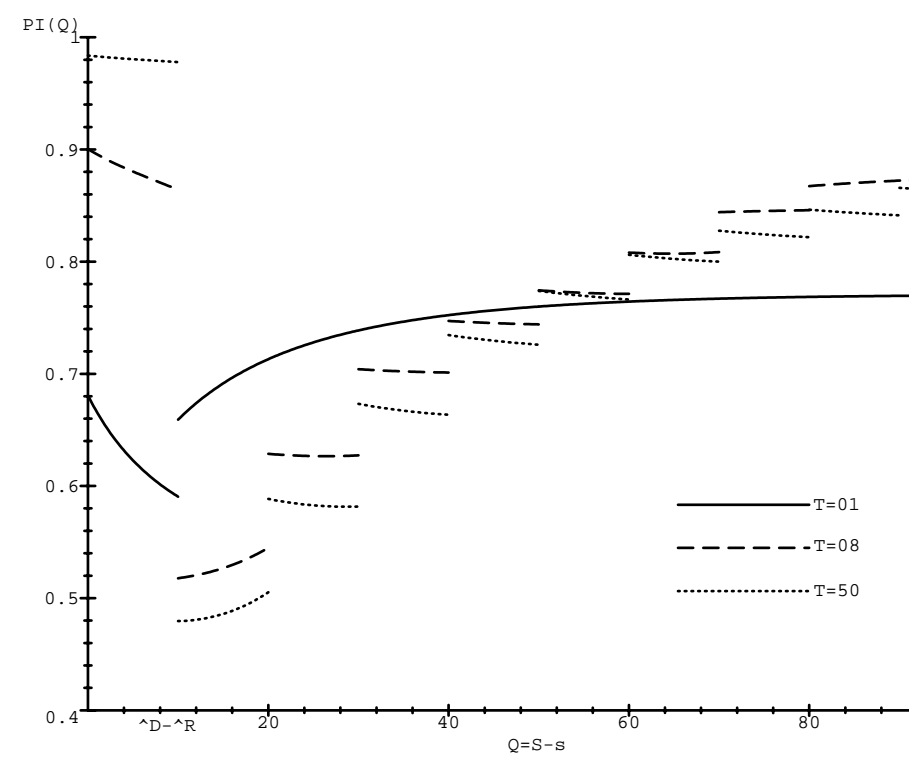

Fig. 4. Stability function in recovery model (for different planning horizons)

where $Q$ corresponds to multiples of the expected (net) demand. For instance, for $T=8$ the stability functions are continuous for $Q>80$, and discontinuous in $Q=10,20, \ldots ., 80$. Moreover, stability reaches (local) minima in the points of discontinuity. In the traditional model it can be shown analytically that stability admits a global minimum at $Q=\hat{D}$ for the short-term case, and at $Q=2 \hat{D}$ for 
$T>1$. Note that this can also be observed in Figure 3. For the recovery model in Figure 4 minimal stability is reached for values of $Q$ approaching $\hat{N}$ either from below $(T=1)$ or from above $(T>1)$.

In general, we have found that minimal stability is always attained for lot sizes in the interval $(\hat{N}, 2 \hat{N})$. For example, for $\hat{D}=30, \hat{R}=10$ the minimum is attained for $Q=29.5$ with a stability level of $51.1 \%$. It is never reached for lot sizes which are equal to or larger than twice as big as the expected net demand. For an explanation consider the sequence of setups for $\hat{N}<Q \leq 2 \hat{N}$. After the first planned setup we find a planned production order for every second period, i.e in cycle $t$ setups in periods $t+\tau+2 n$ (where $\tau$ depends on the initial stock), and in cycle $t+1$ setups occur in $t+\theta+2 n$ (with $n \in \mathbb{I}$ ). Thus for $\tau=\theta$ we find identical order decisions in both planning periods. In all other cases the planned setups deviate significantly and in the worst case may differ in each period. Therefore, the stability level for $l=2$ is rather low. For larger lot sizes there are no planned order sequences that differ in all periods, since for increasing $Q$ the number of periods with no setups increases in both planning cycles.

Considering the impact of $T$ on $\pi$ in Figures 3 and 4 we find that stability increases with a growing planning horizon if $Q<\hat{D}$, or $Q<\hat{N}$, respectively. For larger lot sizes there are some cases where stability increases for a longer planning horizon in the traditional model, but in general it is reduced (see also [4]). This general tendency can also be observed in the recovery model for sufficiently large planning horizons. If we consider the shape of the stability function for $T=8$ and $T=50$ in Figure 4, then we find that an increase of the planning horizon leads to a lower stability level for all lot sizes $Q>\hat{N}$. However, the shape of the short-term stability differs significantly for the traditional model and the recovery system: in the traditional model stability is maximal for $T=1$, whereas in the recovery model $T=1$ leads to a rather small and, for large values of $Q$, even minimal stability level.

\subsection{The impact of the return volume on planning stability}

Let us now consider the impact of the expected returns on stability. To this end, Figure 5 shows planning stability as a function of $\hat{R}$. Table 2 summarizes the corresponding parameter settings. The result is somewhat counterintuitive. For small return rates we find that planning stability decreases as $\hat{R}$ increases. This is what one may expect since the return intensity increases the variability of the net demand per period. However, for large $\hat{R}$ planning stability increases as the return rate increases. For $\hat{R}$ close to $\hat{D}$ stability even converges to 1 . To understand this effect it is useful to consider the expected stock level (see Fig. 6). For high return rates the average stock level increases exponentially in $\hat{R}$, similar to the expected queue length in a single server queue. The high stock level implies that there are very few production orders scheduled, and hence that the system is very 'stable' from a setup perspective. In this way, product returns can increase planning stability, however at the expense of high stock levels. Note that this result holds for arbitrary distributions of demand and returns, because the average stock increases in general 


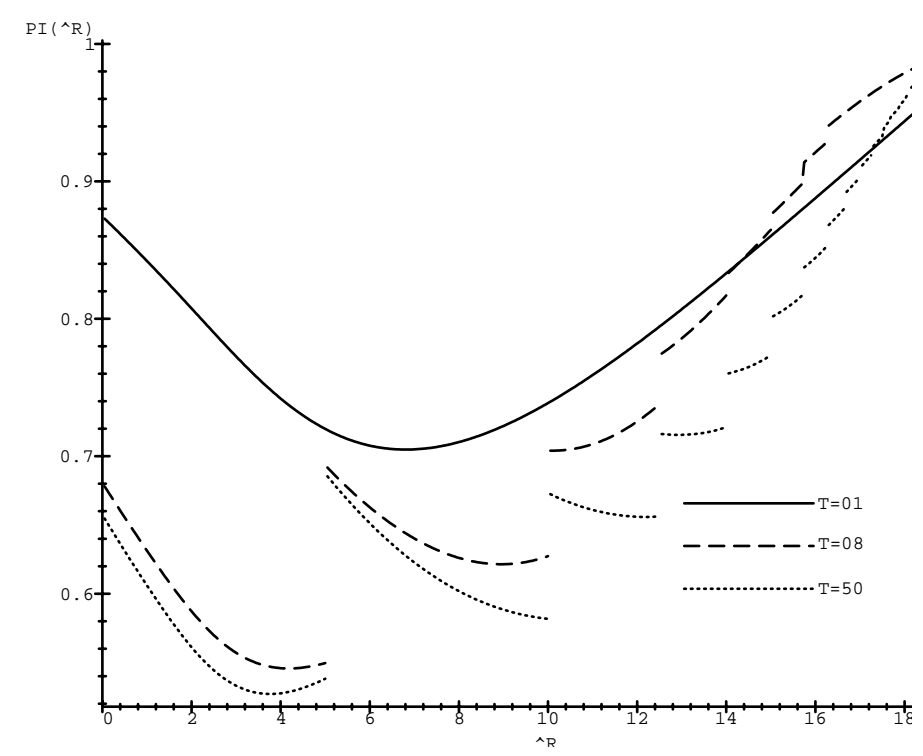

Fig. 5. Stability function for different return rates

Table 2. Parameter settings Figures 5 and 6

\begin{tabular}{c|c|c}
\hline$Q$ & $\hat{D}$ & $\mathrm{~T}$ \\
\hline 30 & 20 & $1,8,50$ \\
\hline
\end{tabular}

for large return rates, i.e. $\bar{R} \rightarrow \bar{D}$.

In Figure 5 we observe for the short-term perspective that the stability level in the recovery model (with $\hat{R}>0$ ) is larger than stability in the traditional model (with $\hat{R}=0$ ) for return/demand-ratios larger than $75 \%$. For smaller return intensities stability is lower than in the case of no product returns. For larger planning horizons we find the same relation already for smaller return ratios. For $T=8$ and $T=50$ stability in the recovery model is larger if $\hat{R} / \hat{D}>50 \%$.

In Section 4 in (8) we have given an indication of the influence of returns on planning updates. We have seen that the shift of the order sequence during a planning update is increasing in both the expected value and the variance of the returns per period. Notice that (8) only considers the difference between $\tau$ and $\theta$. In Figure 5 we find that stability increases with rising $\hat{R}$. Though $(8)$ indicates that the expected difference between both planning cycles increases. However the absolute values of $\tau$ and $\theta$ also increase with rising return rate. Therefore, we find a long period with non-setup decisions in both planning cycles. This finally leads to a high level of stability for large return rates, even though (8) may suggest the opposite.

We have also examined the adjustment of $Q$ as a function of the return rate. To this end, we have used an EOQ-approximation to set the value for $Q$ depending 


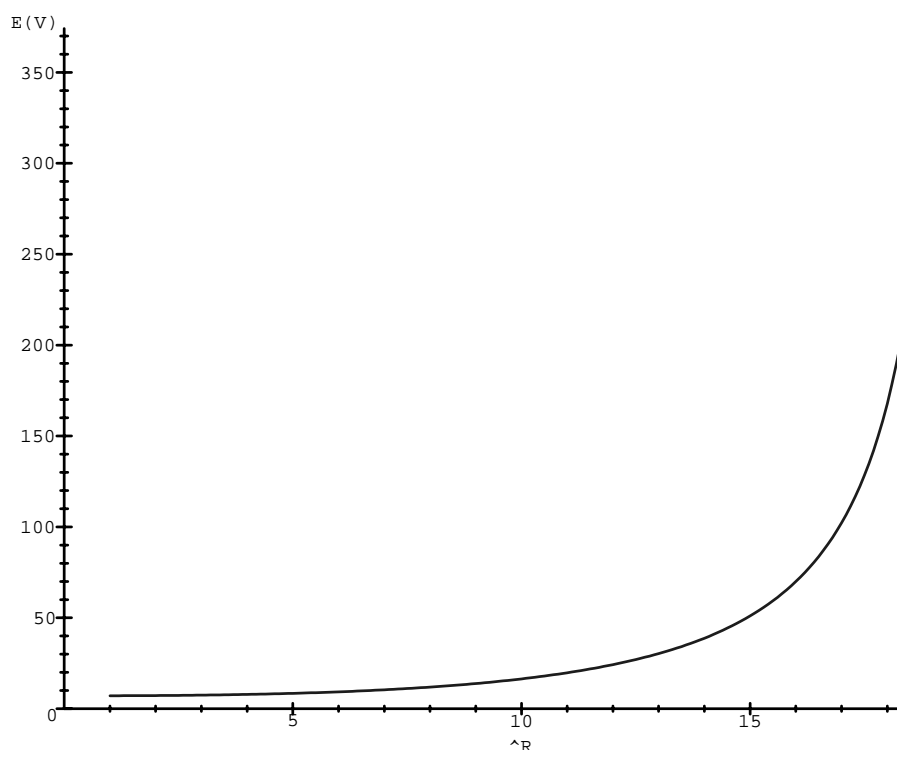

Fig. 6. Average inventory position $\mathbb{E}[V]=\mathbb{E}[Y-s]$

on the return volume. However, this adjustment does not change the shape of the stability function as shown in Figure 5 significantly. We remark that similar results have been reported for traditional inventory models (see Jensen [12]).

Finally, we have varied $Q$ and $\hat{R}$ simultaneously. Figure 7 shows the shape of the stability function for $T=8, \hat{D}=30, Q=0,5,10, \ldots, 40$ and $\hat{R}=0, \ldots, 29.9$. For the sake of clarity the function is plotted in the form of continuous lines across the discontinuities for larger lotsizes. The 'jumps' are clearly visible, in particular for moderate return rates. For example, for $Q=20$ the stability function is discontinuous at $\hat{R}=10,20,23 \frac{1}{3}, 25,26,26 \frac{2}{3}, \ldots$ As discussed above, one observes again that $\pi$ tends to decrease in $\hat{R}$ for $Q \leq \hat{D}-\hat{R}$ and to increase otherwise. Finally, it is worth noting that the case $Q=0$ corresponds with a simple $S$-policy as in [5], for which the stability function is continuous.

\section{Conclusions}

In this paper we have addressed planning stability of production and remanufacturing setups in a basic product recovery system. We have derived both analytic and numerical results.

First of all, we have shown that the sequence of planned orders generated in each planning cycle has largely the same structure as in a traditional inventory system. The main difference concerns a possibly larger initial stock level in the product recovery environment. 


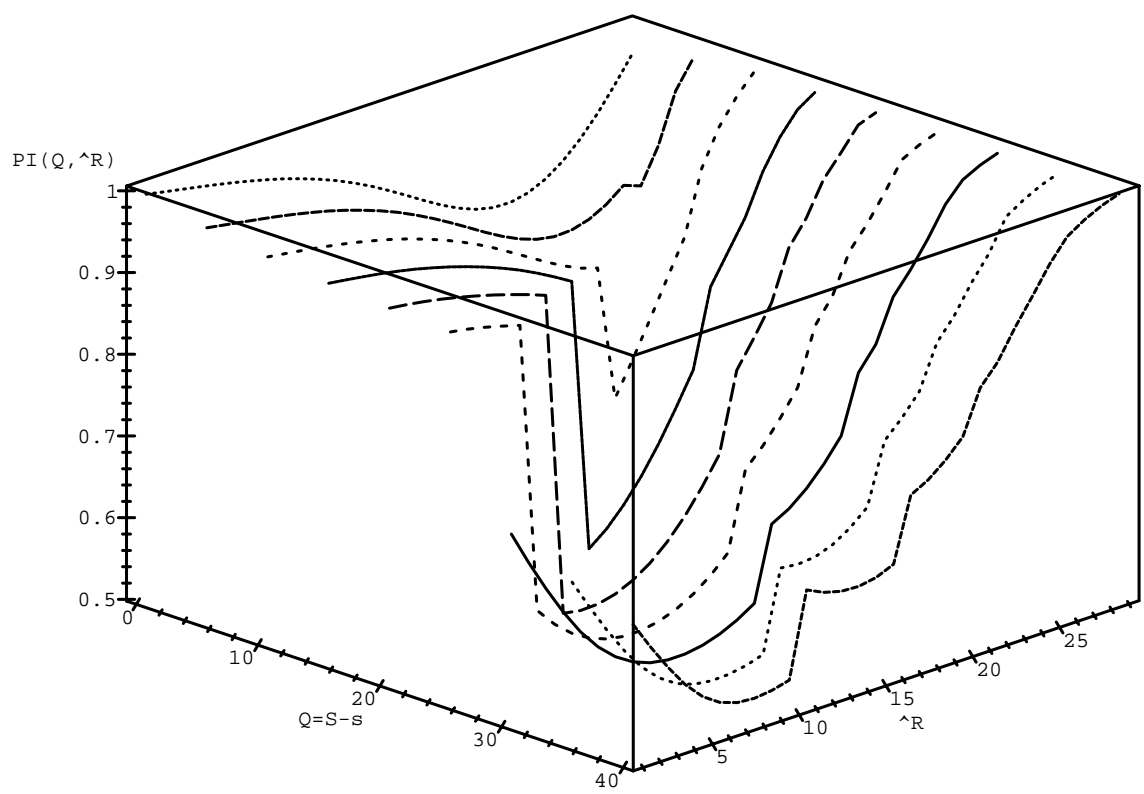

Fig. 7. Stability function for different lot sizes and return rates $(\hat{D}=30$ and $T=8)$

Furthermore, we have analyzed the impact of the product return flow on planning stability, specifically. We have shown that for moderate return rates stability tends to be decreasing in the return rate. This is in line with results from conventional inventory models where stability has been shown to be decreasing in the coefficient of variation of demand. However, for large return rates we have observed a tradeoff between growing demand variability and a rising stock level. Somewhat counterintuitively high return rates, implying high net demand variability, therefore result in high planning stability - though at the expense of high stock levels.

The impact of other system parameters on stability has been found to be largely in parallel with conventional inventory models. In particular, setup stability is not affected by the value of the reorder point $s$, but only by the minimum lot size $Q=S-s$ and the length of the stability horizon $T$.

Depending on the relation between the expected time between ordering and the planning horizon, the stability function is either continuous in the lot size parameter or has cyclical patterns with peaks of nervousness for lot sizes equal to a multiple of the forecasted net demand per period. Furthermore, stability admits a minimum for a reorder quantity equal to the forecasted net demand (if $T=1$ ), or for a lot size in the interval $(\hat{N}, 2 \hat{N})$ (if $T>1$ ). The latter result slightly differs from the case of a traditional $(s, S)$-inventory model where long-term stability always admits its minimum for a lot size equal to twice the expected demand per period.

Finally, the planning horizon influences planning stability as follows. For small lot sizes, i.e. $Q \leq \hat{N}$, the level of stability is increasing in the planning horizon. For larger lot sizes the picture is less clear. In general, we found stability to be 
decreasing in the planning horizon, except for the case of a very short horizon, where stability turns out to be rather low as compared to a traditional model, in particular for larger lot sizes.

As discussed before, the recovery system considered in this paper is a very basic one. The analysis may be extended in several directions. First of all, we have assumed all returned products to be recovered immediately. Alternative models have been proposed in literature that encompass an additional stock of returned products (see, e.g., $[14,9,17,18]$ ). In this case, the timing of the recovery process may be adjusted according to lot sizing or postponement considerations. In general, one does not find an easily implementable optimal policy structure for these models, due to a growing dimensionality of the underlying Markov processes. $(s, Q)$-heuristics have been proposed for controlling both the production and the remanufacturing process. Postponing the recovery process also influences planning stability. On the one hand, nervousness in the recovery process itself can be expected to behave as in a conventional $(s, Q)$-system rather than an order up-to- $S$ system as in our model above. Furthermore, one may expect nervousness in the production process to increase, since the output of the recovery process becomes more irregular. A more thorough analysis of these effects appears worthwhile.

Second, we have not considered the option of disposing of excessive returns. In contrast, some of the aforementioned models in literature include such a disposal option. For example, a mixed 'order-upto-dispose-downto' has shown to be optimal under certain conditions (see [15,9]). In general, there is again no simple optimal policy structure. Since we have seen that the unboundedness of the stock level plays an important role for determining planning stability in our model one may expect that introducing a disposal option has a significant impact on stability. In particular, stability for high return rates can be expected to decrease, since disposal reduces the 'dampening' effect of a rising stock level. We refer to Heisig [6] for initial results on this issue.

Third, we have assumed demand and returns to be independent processes. In many practical situations returns may rather depend on previous demand. In this case, assuming independence between demand and returns essentially means to ignore some part of the information that is available on future returns. The impact of this omission very much depends on the specific context. In general, one may expect demand information to be important for returns forecasting in cases of highly irregular demand (e.g. due to seasonal peaks) and rather short market sojourn times. Durable products such as electronics equipment or cars are typically only returned after several years. In this case, variability in the market sojourn time is large compared to the inventory system's average time between ordering. Therefore, exploiting the correlation between demand and returns may primarily be useful for updating the return rate during a product lifecycle rather than for controlling individual orders. Therefore, we conclude that assuming independent demand and returns is at least a good approximation in many cases, which yields a simple and easily implementable control policy.

In our numerical example we have assumed demand and returns to be exponentially distributed, in order to allow for easily tractable expressions. We recall that results for traditional inventory models suggest the specific form of the demand 
distribution to have a fairly limited impact on the properties of the stability function (see [1], [4], [12]). While a growing coefficient of variation, in general, results in a higher overall level of nervousness qualitative results remain largely unchanged. We expect a similar behaviour for product recovery systems. Nevertheless, addressing alternative net demand distributions in future research may be worthwhile.

Future research may also address alternative stability aspects like quantityoriented stability or additional uncertainties such as stochastic lead times. However, since stability analysis under these conditions turns out to be fairly intractable for traditional inventory models one may expect similar difficulties for the case of recovery systems.

To sum up, system nervousness may be a reason for additional considerations in determining inventory control rules and control parameters. Similar to service level constraints, the additional factor of planning stability can be included in inventory control by using analogous stability constraints. The results in this paper illustrate how lot sizing in a product recovery system may be adjusted to take such managerial constraints into account.

\section{Appendix A. Stationary inventory distribution}

In this appendix we determine an explicit expression for the stationary distribution of the net stock level for the case of exponentially distributed demand and returns. Hence, let $D_{t}$ and $R_{t}$ be exponentially distributed for each $t$ with parameters $\lambda$ and $\mu$, respectively. Note that our assumption $\mathbb{E}[N]>0$ implies $\lambda<\mu$. Moreover, recall that $V_{t}=Y_{t}-s$ where $Y_{t}$ denotes the net stock at the beginning of period $t$ after remanufacturing and production as defined in Section 2. As discussed in Section 2, the existence and uniqueness of a stationary distribution $F_{V}$ of the process $\left(V_{t}\right)$ on $[0, \infty)$ follow from the fact that $\left(V_{t}\right)$ is an ergodic random walk (see [3]). Hence, it suffices to determine the explicit form of $F_{V}$. We do so by considering the stationarity equation of the process $\left(V_{t}\right)$.

As before, let $\Phi(n)=\mathbb{P}\{N \leq n\}$ denote the distribution of the net demand in an arbitrary period and $\phi(n)$ the corresponding density function. Assuming exponential distributions for $D_{t}$ and $R_{t}$ yields

$$
\phi(n)=\left\{\begin{array}{lll}
\frac{\mu \lambda}{\mu+\lambda} e^{-\lambda n} & \text { for } & n \geq 0 \\
\frac{\mu \lambda}{\mu+\lambda} e^{\mu n} & \text { for } & n<0
\end{array}\right.
$$

and

$$
\Phi(n)=\left\{\begin{array}{lll}
1-\frac{\mu}{\mu+\lambda} e^{-\lambda n} & \text { for } & n \geq 0 \\
\frac{\lambda}{\mu+\lambda} e^{\mu n} & \text { for } & n<0 .
\end{array}\right.
$$

Furthermore, for $x \geq 0$ and $v \in \mathbb{R}$ let

$$
q(x, v)=\mathbb{P}\left\{V_{t+1} \leq v \mid V_{t}=x\right\} .
$$


Inserting the transition probabilities of $V_{t}$ yields

$$
q(x, v)=\int_{0}^{v} \phi(x-u) d u+1_{v \geq Q}(1-\Phi(x)),
$$

where $1_{v \geq Q}$ denotes the indicator function of the condition $v \geq Q$. Stationarity of $F_{V}$ with respect to $\left(V_{t}\right)$ implies

$$
F_{V}(v)=\int_{0}^{\infty} q(x, v) F_{V}(d x) \forall v \in \mathbb{R} .
$$

Suppose that $F_{V}$ can be written in the form

$$
F_{V}(v)=\int_{0}^{v} f_{V}(x) d x+1_{v \geq Q} p_{Q},
$$

where the first term denotes an absolutely continuous part and the second term denotes a probability mass at $v=Q$. Then (A.2) is equivalent with

$$
\begin{aligned}
\int_{0}^{v} f_{V}(x) d x+\mathbb{1}_{v \geq Q} p_{Q}= & \int_{0}^{\infty}\left[\int_{0}^{v} \phi(u-x) d x+\mathbb{1}_{v \geq Q}(1-\Phi(u))\right] f_{V}(u) d u \\
& +p_{Q}\left[\int_{0}^{v} \phi(Q-x) d x+\mathbb{1}_{v \geq Q}(1-\Phi(Q))\right] \forall v \in \mathbb{R} .
\end{aligned}
$$

Letting $v \rightarrow Q$ yields

$$
p_{Q}=\int_{0}^{\infty}(1-\Phi(u)) f_{V}(u) d u+p_{Q}(1-\Phi(Q))
$$

and therefore

$$
\int_{0}^{v} f_{V}(x) d x=\int_{0}^{v}\left[\int_{0}^{\infty} \phi(u-x) f_{V}(u) d u+p_{Q} \phi(Q-x)\right] d x \quad \forall v \in \mathbb{R},
$$

and further

$$
f_{V}(x)=\int_{0}^{\infty} \phi(u-x) f_{V}(u) d u+p_{Q} \phi(Q-x) \forall x \in \mathbb{R} .
$$

Solving (A.3) for $p_{Q}$ and inserting into (A.4) yields the following integral equation for $f_{V}$

$$
f_{V}(x)=\int_{0}^{\infty}\left(\phi(u-x)+\frac{\phi(Q-x)}{\Phi(Q)}(1-\Phi(u)) f_{V}(u) d u \quad \forall x \in \mathbb{R} .\right.
$$

We can rewrite this condition further by using (A.1). To this end we must distinguish two cases, namely $x \leq Q$ and $x>Q$. Let us first assume that $x \leq Q$. Then (A.5) yields

$$
\begin{aligned}
f_{V}(x)=\frac{\mu \lambda}{\mu+\lambda} & {\left[e^{-\mu x} \int_{0}^{x} e^{\mu u} f_{V}(u) d u\right.} \\
& +e^{\lambda x} \int_{x}^{\infty} e^{-\lambda u} f_{V}(u) d u \\
& \left.+e^{\lambda x} \int_{0}^{\infty} e^{-\lambda Q} \frac{\mu e^{-\lambda u}}{\lambda+\mu\left(1-e^{-\lambda Q}\right)} f_{V}(u) d u\right] \quad \forall x \leq Q .
\end{aligned}
$$


Repeatedly differentiating this equation leads to the following differential equation for $f_{V}$

$$
f_{V}^{\prime \prime \prime}(x)=(\lambda-\mu) f_{V}^{\prime \prime}(x) \quad \forall x \leq Q
$$

Moreover, we find that inserting (A.1) into (A.5) for $x>Q$ leads to the same differential equation. Hence (A.7) must hold for all $x \geq 0$. Clearly, the general solution of (A.7) has the form

$$
f_{V}(x)=a+b x+c e^{(\lambda-\mu) x}
$$

with some constants $a, b$ and $c$. Since $\lim _{x \rightarrow \infty} f_{V}(x)$ must equal zero we get that $a=b=0$. Moreover, using (A.4) and (A.3) and the fact that $\int_{0}^{\infty} f_{V}(x) d x+p_{Q}$ must equal one yields the value of the constant $c$. In this way we finally get

$$
F_{V}(v)=\int_{-\infty}^{v} f_{V}(x) d x+1_{v \geq Q} p_{Q},
$$

with $\quad p_{Q}=\frac{\mu-\lambda}{\mu\left(2-e^{-\lambda Q}\right)}$

and $f_{V}(v)= \begin{cases}0 & \text { for } \quad v<0 \\ c e^{-(\mu-\lambda) v} & \text { for } \quad v \geq 0\end{cases}$

where $c=p_{Q}\left[\lambda+\mu\left(1-e^{-\lambda Q}\right)\right]$.

\section{B Stability function}

In this section we compute an explicit expression for the expected planning stability $\pi$ for the case of exponentially distributed demand and returns. Recall from (9) that we have the general expression

$$
\begin{gathered}
\pi=\sum_{\tau=1}^{\infty} \int_{((\tau-1) \hat{N}, \tau \hat{N}]}\left[\sum_{\theta=1}^{\infty} \beta_{\theta}^{\tau} \int_{v-(\theta-1) \hat{N}}^{v-(\theta-2) \hat{N}} \phi(n) d n\right. \\
\left.+\beta_{1}^{\tau} \int_{v+\hat{N}}^{\infty} \phi(n) d n\right] d F_{V}(v)
\end{gathered}
$$

Using the fact that $\beta_{\theta}^{T+k}=\beta_{\theta}^{T+1}$ for all $k \geq 1$ and all $\theta$ (and analogous for $\theta \geq T+1$ ) and taking into account the form of the stationary distribution of $V$ 
derived in Appendix A we can rewrite $\pi$ as follows.

$$
\begin{gathered}
\pi=\sum_{\tau=1}^{T} \int_{(\tau-1) \hat{N}}^{\tau \hat{N}}\left[\beta_{1}^{\tau} \int_{v}^{\infty} \phi(n) d n+\sum_{\theta=2}^{T+1} \beta_{\theta}^{\tau} \int_{v-(\theta-1) \hat{N}}^{v-(\theta-2) \hat{N}} \phi(n) d n\right. \\
\left.\quad+\beta_{T+1}^{\tau} \int_{-\infty}^{v-T \hat{N}} \phi(n) d n\right] f_{V}(v) d v \\
+\quad \int_{T \hat{N}}^{\infty}\left[\beta_{1}^{T+1} \int_{v}^{\infty} \phi(n) d n+\sum_{\theta=2}^{T+1} \beta_{\theta}^{T+1} \int_{v-(\theta-1) \hat{N}}^{v-(\theta-2) \hat{N}} \phi(n) d n\right. \\
\left.+\beta_{T+1}^{T+1} \int_{-\infty}^{v-T \hat{N}} \phi(n) d n\right] f_{V}(v) d v \\
\quad\left[\beta_{1}^{l} \int_{Q}^{\infty} \phi(n) d n+\sum_{\theta=2}^{T+1} \beta_{\theta}^{l} \int_{Q-(\theta-1) \hat{N}}^{Q-(\theta-2) \hat{N}} \phi(n) d n\right. \\
\left.+\beta_{T+1}^{l} \int_{-\infty}^{Q-T \hat{N}} \phi(n) d n\right]
\end{gathered}
$$

We can evaluate the integrals in this formula by inserting the expressions for the distribution of $N$ and $V$ derived in Appendix A. For $a<b$ we get

$$
\int_{a}^{b} \phi(n) d n= \begin{cases}\frac{\mu}{\mu+\lambda}\left(e^{-\lambda a}-e^{-\lambda b}\right) & \text { for } \quad 0 \leq a<b \\ 1-\frac{\mu}{\mu+\lambda} e^{-\lambda b}-\frac{\lambda}{\mu+\lambda} e^{\mu a} & \text { for } \quad a<0 \leq b \\ \frac{\lambda}{\mu+\lambda}\left(e^{\mu b}-e^{\mu a}\right) & \text { for } \quad a<b<0\end{cases}
$$

and therefore

$$
\begin{aligned}
& \int_{v}^{\infty} \phi(n) d n=\frac{\mu}{\mu+\lambda} e^{-\lambda v} \\
& \int_{v-(\theta-1) \hat{N}}^{v-(\theta-2) \hat{N}} \phi(n) d n \\
& \quad= \begin{cases}\frac{\mu}{\mu+\lambda} e^{-\lambda(v-(\theta-1) \hat{N})}\left(1-e^{-\lambda \hat{N}}\right) & \text { for } \theta \leq v+1 \\
1-\frac{\mu}{\mu+\lambda} e^{-\lambda(v-(\theta-2) \hat{N})}-\frac{\lambda}{\mu+\lambda} e^{\mu(v-(\theta-1) \hat{N})} & \text { for } v+1<\theta \leq v+2 \\
\frac{\lambda}{\mu+\lambda} e^{\mu(v-(\theta-1) \hat{N})}\left(e^{\mu \hat{N}}-1\right) & \text { for } v+2<\theta\end{cases} \\
& \int_{-\infty}^{v-T \hat{N}} \phi(n) d n=\frac{\lambda}{\mu+\lambda} e^{\mu(v-T \hat{N})}
\end{aligned}
$$


and further, setting $\tilde{c}:=c /(\mu+\lambda)$

$$
\begin{aligned}
& \int_{(\tau-1) \hat{N}}^{\tau \hat{N}} \int_{v}^{\infty} \phi(n) f_{V}(v) d n d v=\tilde{c} e^{-\mu \tau \hat{N}}\left(e^{\mu \hat{N}}-1\right) \\
& \int_{(\tau-1) \hat{N}}^{\tau \hat{N}} \int_{v-(\theta-1) \hat{N}}^{v-(\theta-2) \hat{N}} \phi(n) f_{V}(v) d n d v \\
& = \begin{cases}\tilde{c}\left(1-e^{-\lambda \hat{N}}\right)\left(e^{\mu \hat{N}}-1\right) e^{\lambda(\theta-1) \hat{N}} e^{-\mu \tau \hat{N}} & \text { for } \quad \theta \leq \tau \\
\frac{2 \tilde{c}}{\mu-\lambda} e^{-(\mu-\lambda) \tau \hat{N}}\left(\lambda e^{(\mu-\lambda) \hat{N}}+(\mu-\lambda) e^{-\lambda \hat{N}}-\mu\right) & \text { for } \quad \theta=\tau+1 \\
\tilde{c}\left(1-e^{-\lambda \hat{N}}\right)\left(e^{\mu \hat{N}}-1\right) e^{\lambda \tau \hat{N}} e^{-\mu(\theta-1) \hat{N}} & \text { for } \quad \theta \geq \tau+2\end{cases} \\
& \int_{(\tau-1) \hat{N}}^{\tau \hat{N}} \int_{-\infty}^{v-T \hat{N}} \phi(n) f_{V}(v) d n d v=\tilde{c} e^{-\mu T \hat{N}}\left(1-e^{-\lambda \hat{N}}\right) e^{\lambda \tau \hat{N}} \\
& \int_{T \hat{N}}^{\infty} \int_{v}^{\infty} \phi(n) f_{V}(v) d n d v=\tilde{c} e^{-\mu T \hat{N}} \\
& \int_{T \hat{N}}^{\infty} \int_{v-(\theta-1) \hat{N}}^{v-(\theta-2) \hat{N}} \phi(n) f_{V}(v) d n d v=\tilde{c}\left(1-e^{-\lambda \hat{N}}\right) e^{-\mu T \hat{N}} e^{\lambda(\theta-1) \hat{N}} \\
& \int_{T \hat{N}}^{\infty} \int_{-\infty}^{v-T \hat{N}} \phi(n) f_{V}(v) d n d v=\frac{2 \tilde{c} \lambda}{\mu-\lambda} e^{-(\mu-\lambda) T \hat{N}}
\end{aligned}
$$

We can now put all these results together. For ease of notation we set $c_{\mu}:=$ $\left(e^{\mu \hat{N}}-1\right), c_{\lambda}:=\left(1-e^{-\lambda \hat{N}}\right)$, and $c_{T}:=e^{-\mu T \hat{N}}$. We need to distinguish two cases concerning the relation of $l$ and $T$. (Note that the distinction only affects the last term.)

Case A: For $l \leq T$ we have

$$
\begin{aligned}
\pi=\tilde{c}\left\{\sum_{\tau=1}^{T}\right. & {\left[\beta_{1}^{\tau} c_{\mu} e^{-\mu \tau \hat{N}}+\sum_{\theta=2}^{\tau} \beta_{\theta}^{\tau} c_{\mu} c_{\lambda} e^{-(\mu \tau-\lambda(\theta-1)) \hat{N}}\right.} \\
& +\beta_{\tau+1}^{\tau} \frac{2}{\mu-\lambda}\left(\lambda c_{\mu}\left(1-c_{\lambda}\right)-\mu c_{\lambda}\right) e^{-(\mu-\lambda) \tau \hat{N}} \\
& \left.+\sum_{\theta=\tau+2}^{T+1} \beta_{\theta}^{\tau} c_{\mu} c_{\lambda} e^{-(\mu(\theta-1)-\lambda \tau) \hat{N}}+\beta_{T+1}^{\tau} c_{T} c_{\lambda} e^{\lambda \tau \hat{N}}\right]
\end{aligned}
$$




$$
\begin{gathered}
\left.+\beta_{1}^{T+1} c_{T}+\sum_{\theta=2}^{T+1} \beta_{\theta}^{T+1} c_{T} c_{\lambda} e^{\lambda(\theta-1) \hat{N}}+\beta_{T+1}^{T+1} \frac{2 \lambda}{\mu-\lambda} e^{-(\mu-\lambda) T \hat{N}}\right\} \\
+\frac{p_{Q}}{\mu+\lambda}\left[\beta_{1}^{l} \mu e^{-\lambda Q}+\sum_{\theta=2}^{l} \beta_{\theta}^{l} \mu c_{\lambda} e^{-\lambda(Q-(\theta-1) \hat{N})}\right. \\
+\quad \beta_{l+1}^{l}\left(\mu+\lambda-\mu\left(1-c_{\lambda}\right) e^{-\lambda(Q-l \hat{N})}-\lambda e^{\mu(Q-l \hat{N})}\right) \\
\left.+\sum_{\theta=l+2}^{T+1} \beta_{\theta}^{l} \lambda c_{\mu} e^{\mu(Q-(\theta-1) \hat{N})}+\beta_{T+1}^{l} \lambda c_{T} e^{\mu Q}\right]
\end{gathered}
$$

Case B: For $l>T$ we have

$$
\begin{aligned}
\pi= & \tilde{c}\left\{\sum _ { \tau = 1 } ^ { T } \left[\beta_{1}^{\tau} c_{\mu} e^{-\mu \tau \hat{N}}+\sum_{\theta=2}^{\tau} \beta_{\theta}^{\tau} c_{\mu} c_{\lambda} e^{-(\mu \tau-\lambda(\theta-1)) \hat{N}}\right.\right. \\
& +\beta_{\tau+1}^{\tau} \frac{2}{\mu-\lambda}\left(\lambda c_{\mu}\left(1-c_{\lambda}\right)-\mu c_{\lambda}\right) e^{-(\mu-\lambda) \tau \hat{N}} \\
& \left.+\sum_{\theta=\tau+2}^{T+1} \beta_{\theta}^{\tau} c_{\mu} c_{\lambda} e^{-(\mu(\theta-1)-\lambda \tau) \hat{N}}+\beta_{T+1}^{\tau} c_{T} c_{\lambda} e^{\lambda \tau \hat{N}}\right] \\
& \left.+\beta_{1}^{T+1} c_{T}+\sum_{\theta=2}^{T+1} \beta_{\theta}^{T+1} c_{T} c_{\lambda} e^{\lambda(\theta-1) \hat{N}}+\beta_{T+1}^{T+1} \frac{2 \lambda}{\mu-\lambda} e^{-(\mu-\lambda) T \hat{N}}\right\} \\
& +\frac{p_{Q}}{\mu+\lambda}\left[\beta_{1}^{l} \mu e^{-\lambda Q}+\sum_{\theta=2}^{T+1} \beta_{\theta}^{l} \mu c_{\lambda} e^{-\lambda(Q-(\theta-1) \hat{N})}\right. \\
& \left.+\beta_{T+1}^{l}\left(\mu+\lambda-\mu e^{-\lambda(Q-T \hat{N})}\right)\right]
\end{aligned}
$$

\section{References}

1. De Kok AG, Inderfurth K (1997) Nervousness in inventory management: comparison of basic control rules. European Journal of Operational Research 103:55-82

2. Fleischmann M, Bloemhof-Ruwaard J, Dekker R, Van der Laan EA, Van Nunen JAEE, Van Wassenhove LN (1997) Quantitative models for reverse logistics: A review. European Journal of Operations Research 103:1-17

3. Fleischmann M, Kuik R (1998) On optimal inventory control with stochastic item returns. Management Report No. 21, Erasmus University Rotterdam, Rotterdam

4. Heisig G (1998) Planning stability under $(\mathrm{s}, \mathrm{S})$ inventory control rules. OR Spektrum 20:215-228

5. Heisig G (1999) Nervousness of a modified order-up-to-level inventory control rule in product recovery systems. Preprint No. 8, Otto-von-Guericke-University Magdeburg, Faculty of Economics and Management, Magdeburg

6. Heisig G (2000) Planning stability in material requirement planning systems. PhD thesis, Otto-von-Guericke-University Magdeburg, Faculty of Economics and Management, Magdeburg. Chapter 5 (to appear) 
7. Heyman DP (1977) Optimal disposal policies for a single-item inventory system with returns. Naval Research Logistics Quarterly 24:385-405

8. Inderfurth K (1994) Nervousness in inventory control: analytical results. OR Spektrum $16: 113-123$

9. Inderfurth K (1997) Simple optimal replenishment and disposal policies for a product recovery system with leadtimes. OR Spektrum 19:111-122

10. Inderfurth K, Jensen T (1999) Analysis of MRP policies with recovery options. In: Leopold-Wildburger U, Feichtinger G, Kistner KP (eds) Modelling and decisions in economics, pp 189-228. Physica, Heidelberg

11. Jensen T (1993) Measuring and improving planning stability of reorder-point lot-sizing policies. International Journal of Production Economics 30-31:167-178

12. Jensen T (1996) Planungsstabilität in der Material-Logistik. PhD dissertation, Physica, Heidelberg

13. Kadipasaoglu SN, Sridharan SV (1997) Measurement of instability in multi-level MRP systems. International Journal of Production Research 35:713-737

14. Muckstadt JA, Isaac MH (1981) An analysis of single item inventory systems with returns. Naval Research Logistics Quarterly 28:237-254

15. Simpson VP (1978) Optimum solution structure for a repairable inventory problem. Operations Reseaarch 26(2):270-281

16. Thierry M (1997) An analysis of the impact of product recovery management on manufacturing companies. PhD thesis, Erasmus University Rotterdam, Rotterdam

17. Van der Laan EA, Salomon M (1997) Production planning and inventory control with remanufacturing and disposal. European Journal of Operational Research 102: 264-278

18. Van der Laan EA, Salomon M, Dekker R, Van Wassenhove LN (1999) Inventory control in hybrid systems with remanufacturing. Management Science 45(5): 733-747

19. Vollmann TE, Berry WL, Whybark DC (1988) Manufacturing planning and control systems, 2nd edn. Irwin, Homewood, Ill 